\title{
The prediction for development of COVID- 19 in global major epidemic areas through empirical trends in China by utilizing state transition matrix model
}

\author{
Zhong Zheng ${ }^{1 \dagger}$, Ke $\mathrm{Wu}^{1 \dagger}$, Zhixian $\mathrm{Yao}^{1 \dagger}$, Xinyi Zheng ${ }^{2,3+}$, Junhua Zheng ${ }^{1 *}$ and Jian Chen ${ }^{4,5,66^{*}}$
}

\begin{abstract}
Background: Since pneumonia caused by coronavirus disease 2019 (COVID-19) broke out in Wuhan, Hubei province, China, tremendous infected cases has risen all over the world attributed to its high transmissibility. We aimed to mathematically forecast the inflection point (IFP) of new cases in South Korea, Italy, and Iran, utilizing the transcendental model from China.

Methods: Data from reports released by the National Health Commission of the People's Republic of China (Dec 31, 2019 to Mar 5, 2020) and the World Health Organization (Jan 20, 2020 to Mar 5, 2020) were extracted as the training set and the data from Mar 6 to 9 as the validation set. New close contacts, newly confirmed cases, cumulative confirmed cases, non-severe cases, severe cases, critical cases, cured cases, and death were collected and analyzed. We analyzed the data above through the State Transition Matrix model.

Results: The optimistic scenario (non-Hubei model, daily increment rate of $-3.87 \%$ ), the cautiously optimistic scenario (Hubei model, daily increment rate of $-2.20 \%$ ), and the relatively pessimistic scenario (adjustment, daily increment rate of $-1.50 \%$ ) were inferred and modeling from data in China. The IFP of time in South Korea would be Mar 6 to 12, Italy Mar 10 to 24, and Iran Mar 10 to 24. The numbers of cumulative confirmed patients will reach approximately $20 \mathrm{k}$ in South Korea, $209 \mathrm{k}$ in Italy, and $226 \mathrm{k}$ in Iran under fitting scenarios, respectively. However, with the adoption of different diagnosis criteria, the variation of new cases could impose various influences in the predictive model. If that happens, the IFP of increment will be earlier than predicted above.

Conclusion: The end of the pandemic is still inapproachable, and the number of confirmed cases is still escalating. With the augment of data, the world epidemic trend could be further predicted, and it is imperative to consummate the assignment of global medical resources to curb the development of COVID-19.
\end{abstract}

Keywords: Coronavirus disease 2019, Novel coronavirus pneumonia, Prediction, The state transition matrix model, Inflection point

\footnotetext{
* Correspondence: zhengjh0471@sina.com; jian.chen@jhu.edu

${ }^{\dagger}$ Zhong Zheng, Ke Wu, Zhixian Yao and Xinyi Zheng contributed equally to this work.

'Department of Urology, Shanghai General Hospital, Shanghai Jiao Tong University, School of Medicine, Shanghai, China

${ }^{4}$ CreditWise Technology Company Limited, Floor 4-5, Section B, Building 1

Tianfu 5th Ave., Chengdu Hi-tech Zone, Chengdu 610041, China

Full list of author information is available at the end of the article
}

C The Author(s). 2020 Open Access This article is licensed under a Creative Commons Attribution 4.0 International License, which permits use, sharing, adaptation, distribution and reproduction in any medium or format, as long as you give appropriate credit to the original author(s) and the source, provide a link to the Creative Commons licence, and indicate if changes were made. The images or other third party material in this article are included in the article's Creative Commons licence, unless indicated otherwise in a credit line to the material. If material is not included in the article's Creative Commons licence and your intended use is not permitted by statutory regulation or exceeds the permitted use, you will need to obtain permission directly from the copyright holder. To view a copy of this licence, visit http://creativecommons.org/licenses/by/4.0/ The Creative Commons Public Domain Dedication waiver (http://creativecommons.org/publicdomain/zero/1.0/) applies to the data made available in this article, unless otherwise stated in a credit line to the data. 


\section{Background}

Since the first case of novel coronavirus pneumonia (NCP), caused by coronavirus disease 2019 (COVID-19), occurred in Wuhan, Hubei Province, China, the dreadful epidemic broke out during Dec 2019 to Mar 2020 under the pace of Chinese Spring Festival [1]. With the untiring efforts of the people and the selfless dedication of medical staff, a total of 59,897 cured patients were discharged [2]. By 24:00 on Mar 9, China has accumulated a total of 80 , 754 confirmed cases (including 4794 severe cases) and 3136 dead cases [3]. However, in January, when the largescale outbreak in China began, the disease initiated to spread to other parts of the world $[4,5]$. Up to Mar 9, a total of 7382 cases were confirmed in South Korea, 7375 cases in Italy, and 6566 cases in Iran [6].

Similar to another coronavirus $(\mathrm{CoV})-\mathrm{SARS}-\mathrm{CoV}-$ COVID-19 is an RNA virus that contains particular spike proteins conjugating with angiotensin-converting enzyme 2 (ACE2) that widely expressed in different human tissues [7]. However, its doughty transmissibility in the community has a strong correlation to reasons such as long incubation period, mild early symptoms, and the like $[8,9]$. Even though some studies have proved that Remdesivir designed for the Ebola virus may have a promising effect on COVID-19 [10], the kernel strategies for the prevention and treatment of NCP are still effective quarantine as in the case of SARS [11]. After the implementation of strict isolation methods, the most significant goal is to predict the arrival of the peak and inflection point (IFP) of new cases of NCP so that administrative departments can modify current strategies.

Based on the previous data, we analyzed the epidemic situation in Hubei Province [12]. After the validation of the model in different datasets, we were able to analyze the world epidemic trends, and predict the arrival of peaks and IFPs of newly confirmed cases and provide references for NCP prevention and control strategies in various countries.

\section{Method}

\section{Study population, data collection, and analysis}

Data from reports, including medical observation, close contacts, confirmed cases, severe cases, critical cases, cured cases and death data and corresponding information, released by the Health Commission of Hubei Province (HCHP) (Dec 31, 2019 to Feb 8, 2020) were extracted as the training set. Primarily, the arrival of the IFP of new cases and epidemic trends in Hubei were deduced and testified in the validation set, whose data were extracted from HCHP (Feb 9, 2020 to Mar 5, 2020). Subsequently, another training set consisting of the data from the National Health Commission of the People's Republic of China (NHC) and the World Health Organization (WHO) (Jan 20, 2020 to Mar 5, 2020) were established. Eventually, the data, including cumulative confirmed cases, cumulative cured cases, death data and corresponding information, from NHC and WHO (Mar 6 to 9,2020$)$ were collected and constructed the validation set. The data period starts from Dec 31, 2019 to Mar 9, 2020. Data is updated on the daily basis. All data were analyzed using Microsoft Excel (Microsoft Office 2016) and R studio (R Foundation for Statistical Computing, Vienna, Austria). The world epidemic situation was performed using the $n \operatorname{Cov} 2019$ package of $\mathrm{R}$ [13]. Histogram was obtained using the ggplot 2 packages of R.

\section{State transition matrix model}

State transition matrix (STM) modeling is a wellregarded approach widely applied in clinical decision analysis based on computer simulation. For estimating the IFP of newly confirmed cases and the scale of cumulative cases in the globe in subsequent days, we chose the Markov model cohort simulation.

\section{Parameter selection and estimate}

In order to estimate the risk metrics (infectivity, severity, lethality) of the NCP, we build a STM model as showing in the figure (Fig. 1).

We define the states in this model. Medical Observation (MO) is the close contact of confirmed cases and put into medical observation. In the subsequent days, outcome could be any of the three: confirmed cases, discharged without COVID-19 infection, or stay in MO. Discharge (Disc) is a terminal state for a close contact, until he or she becomes another incident of close contact again. Infected is an intermediate state, where the patient becomes a confirmed infected case. The outcome is binary: severe, or non-severe. And the outcome is revealed immediately. Non-Severe Case (NS) is the patient also has three possible outcomes in the next day: cure, severe case, or stay in non-severe case. Severe Case (S), the patient has three possible outcomes in the next day: critical case, non-severe case, or stay in severe case. Critical Case $(\mathrm{Cr})$, the patient has three possible outcomes in the next day: cured case, severe case, or stay in critical case. Cured Case $(\mathrm{Cu})$ and Death $(\mathrm{D})$ are also the terminal states for the patient. So, at any moment, we can identify the close contact or patient's state by utilizing a state vector, defined as the following:

$$
\mathrm{V}=\left[\begin{array}{lllllll}
M O & \text { Disc } & N S & S & \mathrm{Cr} & \mathrm{Cu} & \mathrm{D}
\end{array}\right],
$$

Where each element of the vector (V) stands for one state in the same sequentially arranged order as mentioned above. Please note that the comfirmed itself is not an independent state, since the outcome is revealed instantaneously, so we combine confirmed case with Non-Severe, Severe, and Critical cases. 
State Transition Matrix of NCP process and outcome

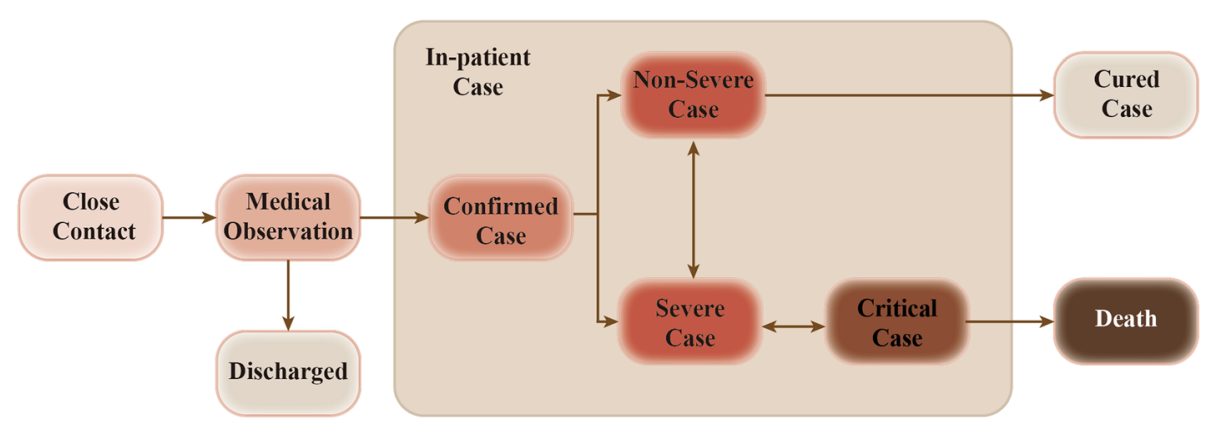

Fig. 1 Process and outcome of the State Transition Matrix establishment when a Close Contact develops into the state of Medical Observation

For each person, the state vector can only have one element with value of 1 , and the other elements all have value of zero. For example, if a patient is currently in

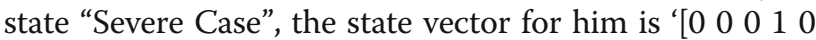
0 0]'. The next day, his state vector could become either

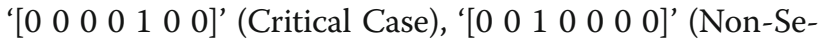
vere Case) or stay the same.

For the sample population, the state vector is defined as the count of people in each state. For example, if there are 100 patients being treated today, out of which 10 are critical, and 90 are severe. The state vector for

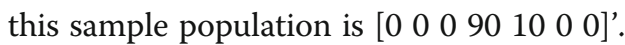

Let's define the STM as the following:

$$
\text { TransMatrix }=\left[t_{i, j}\right]
$$

Where

$$
t_{i, j}=\text { daily transitional probability from state } i \text { to state }
$$

Suppose we have a state vector $\mathrm{V}(\mathrm{t})$ for a sample population at time $t$, how do we predict the state vector $\mathrm{V}(\mathrm{t}+1)$ in the next day?

Apply simple linear algebra, we can get the following equation:

$$
\mathrm{V}(\mathrm{t}+1)=\text { TransMatrix } * \mathrm{~V}(\mathrm{t})
$$

Since the head count of a certain state comes from itself, all other possible transitions into the state (e.g. S has three possible income states, MO, NS, and $\mathrm{Cr}$ ), minus the outcome states (NS, and $\mathrm{Cr}$ ).

If we want to predict for $\mathrm{N}$ period, the equation becomes the following:

$$
\mathrm{V}(\mathrm{t}+\mathrm{N})=\operatorname{TransMatrix}^{N} * \mathrm{~V}(\mathrm{t})
$$

If the population is limited and the transition matrix is stationary, the above formula will be sufficient in predicting all future outcomes. In our case, the population is not fixed, so we need to introduce the additional input into the population: new close contacts (NCC).

Every day, new close contacts are added to the medical observation pool, as people already in the pool will gradually be discharged or confirmed of infection.

$$
\mathrm{MO}(\mathrm{t}+1)=\mathrm{MO}(\mathrm{t})+\mathrm{NCC}(\mathrm{t}+1)-\operatorname{Disc}(\mathrm{t}+1)-\operatorname{Confirmed}(\mathrm{t}+1)
$$

Also, we assume NCC will gradually decay as quarantine measures are put into effect.

$$
\mathrm{NCC}(\mathrm{t}+\mathrm{N})=\mathrm{e}^{\text {Increment } * N} * \mathrm{NCC}(\mathrm{t})
$$

Using this STM model, we will be able to predict when the inflection peak time as well as IFP of newly confirmed cases (the maximum open infection cases) in Hubei Province or non-Hubei will occur. Moreover, after verifying this matrix model in China, it could be $j$ utilized to evaluate the world epidemic development especially in the major epidemic areas.

Although there is an intermediate state during the above hospitalization: severe cases (the new standard is broken down into mild and normal), critical cases (which can also be divided into general critical and critical), due to the lack of intermediate state transfer probability, we combine the entire hospital period into a inpatient state, for the sake of keeping the model simple. This minimizes the need for only the following five parameters.

- Increment of New Close Contacts (NCC), defined as $\ln (\mathrm{NCC}(\mathrm{t}) / \mathrm{NCC}(\mathrm{t}-1))$;

- Discharge Rate from Medical Observation (MO), defined as Discharged(t)/MO(t-1)

- Transitional Probability of Medical Observation - > Confirmed cases, defined as Newly confirmed cases $(\mathrm{t}) / \mathrm{MO}(\mathrm{t}-1)$

- Transitional Probability of Treatment - > Death, defined as New Death Incidents $(\mathrm{t}) /$ Treatment $(\mathrm{t}-\mathrm{1})$ 
Table 1 Scenarios for the prediction of outside China

\begin{tabular}{llll}
\hline Scenario & S1 & S2 & S3 \\
\hline Minimum Inc & $-30 \%$ & $-20 \%$ & $-5 \%$ \\
Daily Inc & $-3.87 \%$ & $-2.20 \%$ & $-1.50 \%$
\end{tabular}

S1: optimistic scenario; S2: cautiously optimistic scenario; S3: relatively pessimistic scenario; Inc. Increment

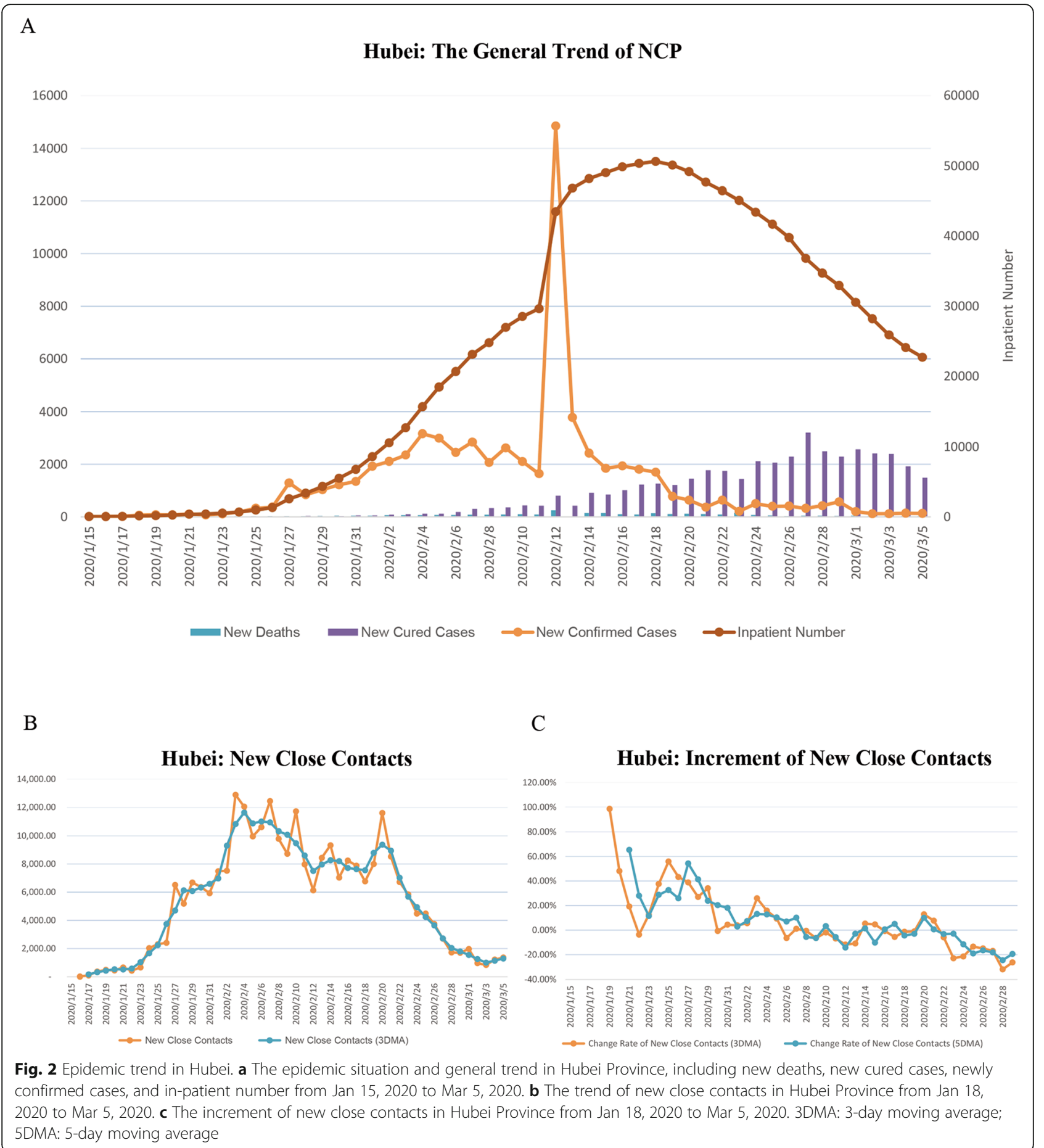


Table 2 Training set and Validation Set of the Epidemic Trend in Hubei Province

\begin{tabular}{|c|c|c|c|c|c|c|c|}
\hline \multirow[b]{2}{*}{ Key Metrics } & \multirow[t]{2}{*}{ Actual } & \multicolumn{6}{|l|}{ Forecast } \\
\hline & & S1 & & S2 & & S3 & \\
\hline Inc of Confirmed Cases & $-9.0 \%$ & $-10 \%$ & $-10 \%$ & $-5 \%$ & $-5 \%$ & $-1 \%$ & $-1 \%$ \\
\hline MO Release Rate & $16.0 \%$ & $17.0 \%$ & $10.50 \%$ & $17.0 \%$ & $10.50 \%$ & $17.0 \%$ & $10.50 \%$ \\
\hline Peak of Active Cases & 50,633 & 39,612 & 47,148 & 44,082 & 55,150 & 62,041 & 85,502 \\
\hline Peak Date & 2020/2/16 & $2020 / 2 / 23$ & $2020 / 2 / 28$ & 2020/3/1 & $2020 / 3 / 7$ & $2020 / 4 / 6$ & $2020 / 4 / 14$ \\
\hline Peak of Severe Cases & 9289 & 5753 & 6845 & 6400 & 8004 & 9000 & 12,402 \\
\hline Peak Date & 2020/2/16 & $2020 / 2 / 23$ & $2020 / 2 / 28$ & $2020 / 3 / 1$ & $2020 / 3 / 7$ & $2020 / 4 / 6$ & $2020 / 4 / 14$ \\
\hline Peak of Critical Cases & 2492 & 1786 & 2124 & 1986 & 2484 & 2793 & 3849 \\
\hline Peak Case & $2020 / 2 / 21$ & $2020 / 2 / 23$ & $2020 / 2 / 28$ & $2020 / 3 / 1$ & $2020 / 3 / 7$ & $2020 / 4 / 6$ & $2020 / 4 / 14$ \\
\hline Total Cases at Feb. End & 66,907 & 54,189 & 64,064 & 60,192 & 71,596 & 68,045 & 81,284 \\
\hline
\end{tabular}

The actual data were extracted from HCHP, and the forecast data in the three scenarios were deduced by the STM model based on the data before Feb 9, 2020. S1: optimistic scenario; S2: cautiously optimistic scenario; S3: relatively pessimistic scenario; MO Medical observation, Inc. Increment

- Transitional Probability of Treatment - > Cured, defined as New Cured Incidents (t) / Treatment $(\mathrm{t}-1)$

In order to estimate the count of open non-severe cases, severe cases, and critical cases, we need three more parameters:
- Ratio of Non-Severe Cases

- Ratio of Severe Cases

- Ratio of Critical Cases

Scenario setup and prediction

After validation of the STM model in Hubei Province, we set up three different scenarios derived from China

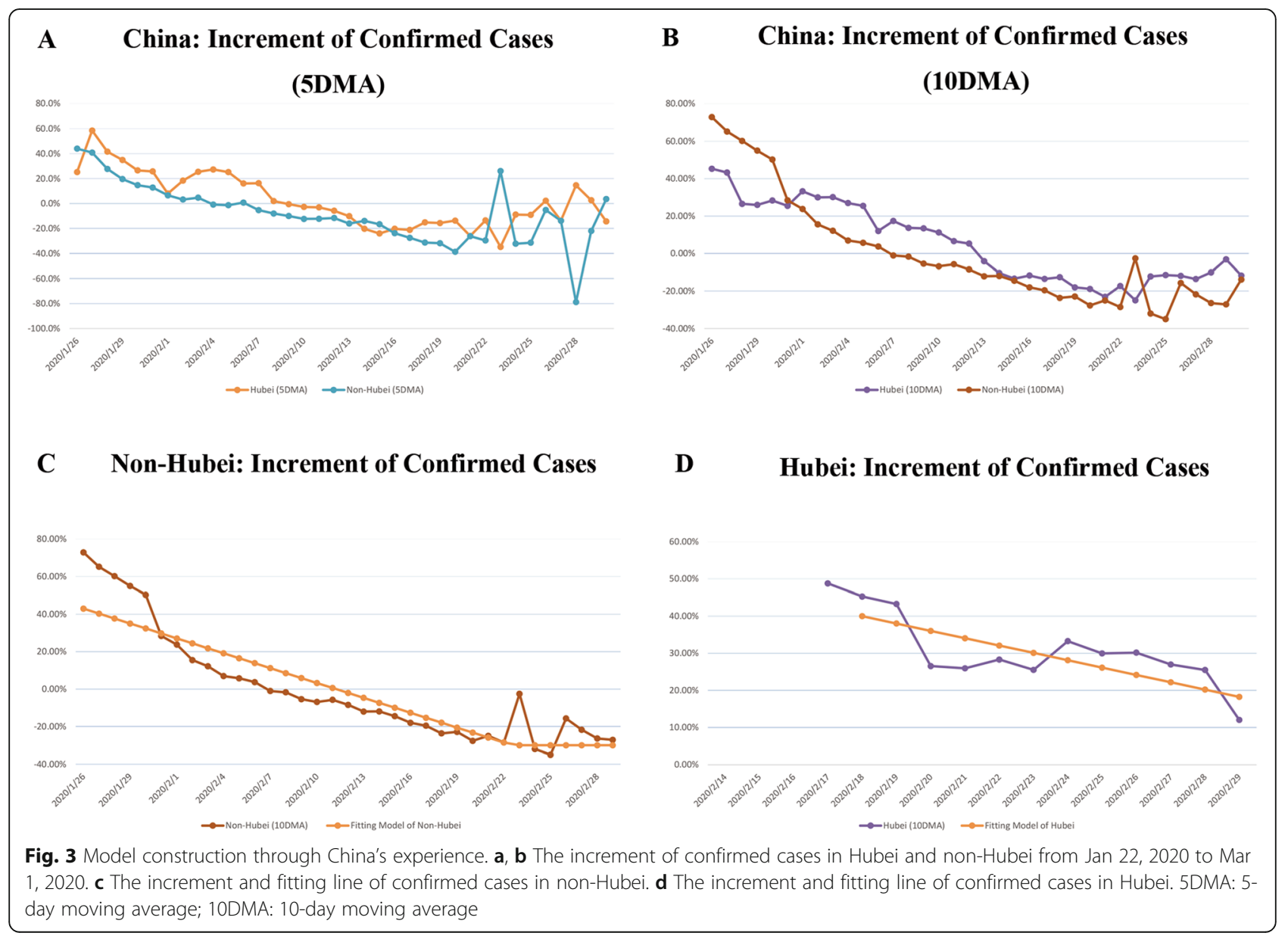


for matching and fitting the major epidemic areas comprising South Korea, Italy, and Iran, in order to control for model error, including optimistic scenario, cautiously optimistic scenario, and relatively pessimistic scenario (Table 1).

\section{Results}

The situation of Hubei Province, China, and the historical prediction model verification of Hubei Province in the beginning of march

According to the data of NHC [14], as of Mar 5, there were 67,592 cumulative confirmed cases, 41,966 cumulative cured cases, 126 newly confirmed cases, 29 new deaths, and 1478 new cured cases, and 19,758 in-patient cases in Hubei Province (Fig. 2a). The number of new close contacts in Hubei Province has gradually decreased, and the cumulative number of close contacts is currently 271,959 (Fig. 2b). The increment of new close contacts has crossed the IFP (Fig. 2c). Based on data from Dec 31, 2019 to Feb 8, 2020 in Hubei Province, we built a prediction model through the STM model, and the cautiously optimistic scenario could consummately predict the arrival of the IFP and several peak dates in Hubei (Table 2), which undoubtedly validate the predictive efficacy of this mathematic model.

\section{Epidemic situation in training set and the epidemic trend fitting model}

As of Mar 5, there were 23,784 confirmed cases, 53,726 cumulative cured cases, 3042 cumulative deaths, 80,552 cumulative confirmed cases, and 670,854 cumulative close contacts in China. Through the analysis, the 5-day moving average (5DMA) and 10-day moving average (10DMA) increment of the confirmed case in Hubei and non-Hubei suggested that the IFP in China was from Feb 6 to Feb 13 (Fig. 3a and b).

Applying the STM model again to establish a 10DMA increment of confirmed cases model in non-Hubei, the fitting line of the trend in non-Hubei could be obtained, which is

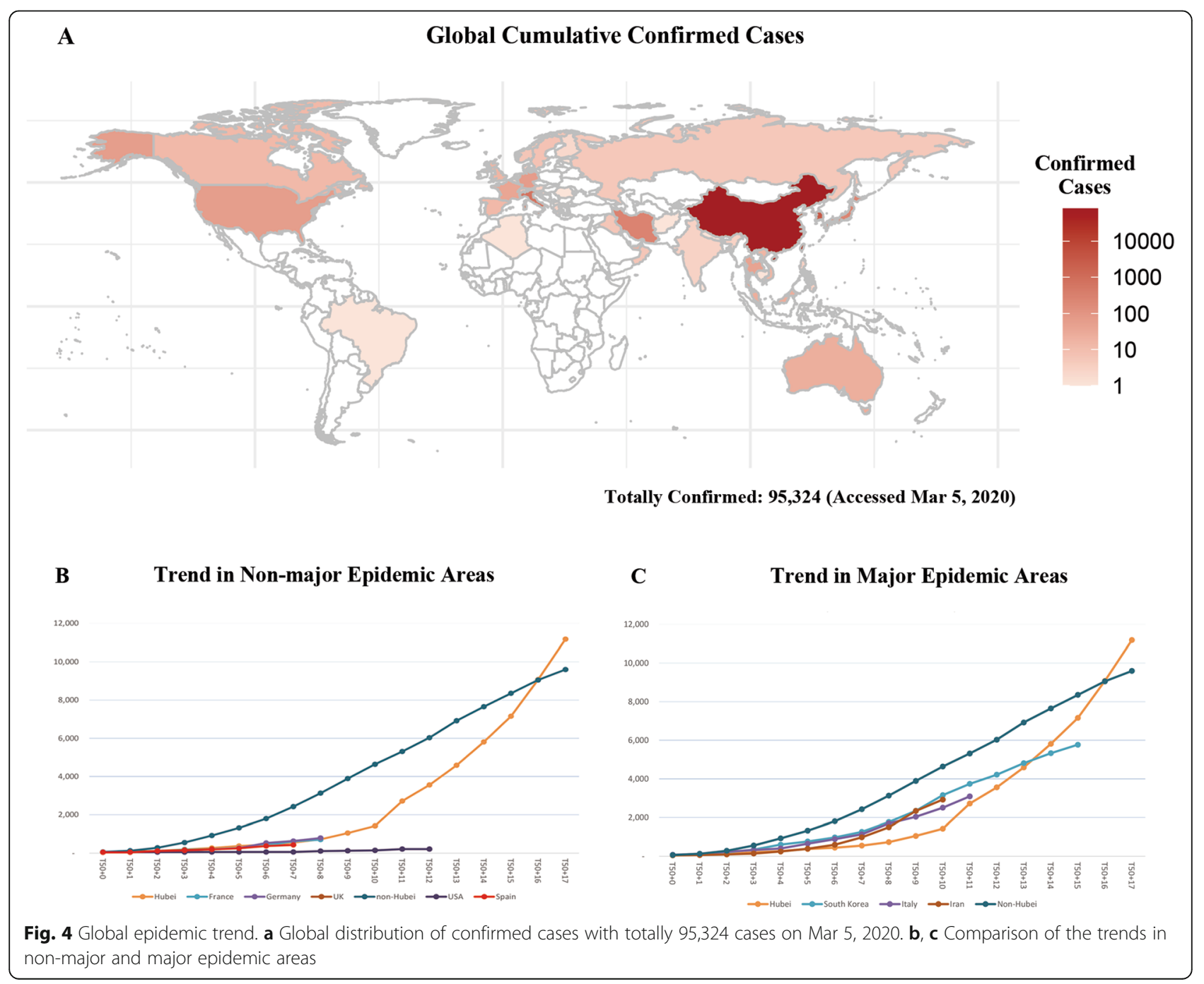




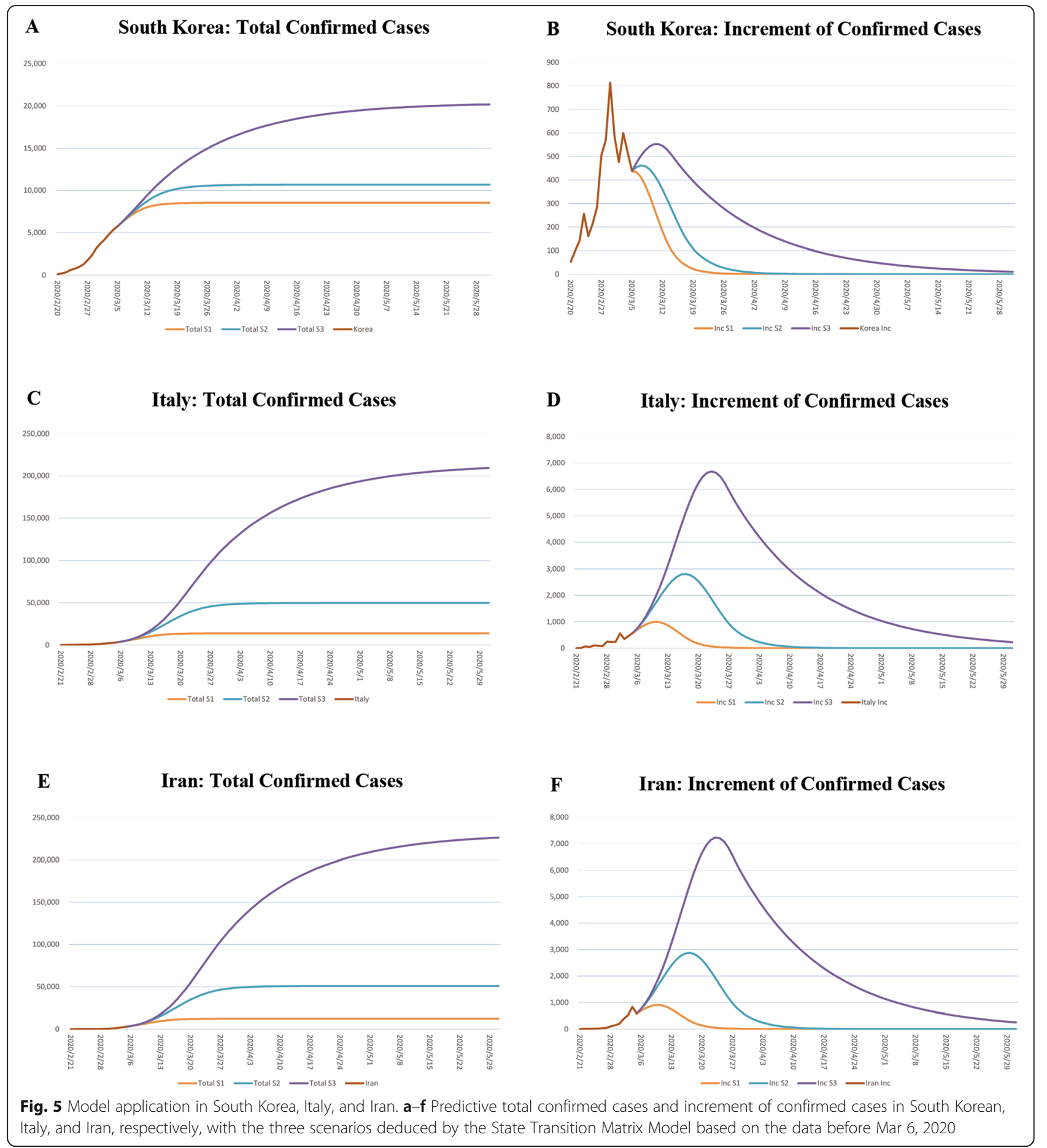

$y=-0.0387 x+1696.2\left(\mathrm{R}^{2}=0.883\right)$

(Fig. 3c).

Similarly, in Hubei, the fitting line is

$$
y=-0.022 x+965.69\left(R^{2}=0.9096\right)
$$

(Fig. 3d).
According to the derivatives taken from fitting lines, the epidemic trend in non-Hubei was set as an optimistic scenario with increment of $-3.87 \%$, and the epidemic trend in Hubei as a cautiously optimistic scenario with increment of $-2.20 \%$, and set a relatively pessimistic scenario with increment of $-1.50 \%$ (Table 1), which could forecast the situation outside China. 
Table 3 Training Set and Validation Set of the Epidemic Trends in the Major Epidemic Areas

\begin{tabular}{|c|c|c|c|c|c|c|c|c|c|c|c|c|}
\hline \multirow{3}{*}{$\begin{array}{l}\text { Countries } \\
\text { Date }\end{array}$} & \multicolumn{4}{|c|}{ South Korea } & \multicolumn{4}{|l|}{ Italy } & \multicolumn{4}{|l|}{ Iran } \\
\hline & \multirow[t]{2}{*}{ Actual } & \multicolumn{3}{|c|}{ Forecast } & \multirow[t]{2}{*}{ Actual } & \multicolumn{3}{|c|}{ Forecast } & \multirow[t]{2}{*}{ Actual } & \multicolumn{3}{|c|}{ Forecast } \\
\hline & & S1 & $\mathrm{S} 2$ & S3 & & S1 & S2 & S3 & & S1 & S2 & S3 \\
\hline $2020 / 3 / 5$ & 5766 & 5766 & 5766 & 5766 & 3089 & 3089 & 3089 & 3089 & 2922 & 2922 & 2922 & 2922 \\
\hline $2020 / 3 / 6$ & 6284 & 6199 & 6221 & 6239 & 3858 & 3794 & 3844 & 3852 & 3513 & 3613 & 3678 & 3687 \\
\hline $2020 / 3 / 7$ & 6767 & 6612 & 6683 & 6741 & 4636 & 4609 & 4795 & 4830 & 4747 & 4396 & 4630 & 4672 \\
\hline $2020 / 3 / 8$ & 7134 & 6990 & 7142 & 7268 & 5883 & 5515 & 5965 & 6064 & 5823 & 5250 & 5806 & 5921 \\
\hline 2020/3/9 & 7382 & 7323 & 7589 & 7811 & 7375 & 6485 & 7374 & 7598 & 6566 & 6147 & 7224 & 7480 \\
\hline
\end{tabular}

S1: optimistic scenario; S2: cautiously optimistic scenario; S3: relatively pessimistic scenario; Inc.: increment

\section{International epidemic situation and prediction}

Data from WHO shows that there were 2232 new cases worldwide on Mar 5, the cumulative number of confirmed cases reached 95,324, and a total of 85 countries have suffered this epidemic (Fig. 4a) [6]. Starting from the cumulative 50 confirmed cases (T50), the cumulative confirmed case trends were compared in different countries with China, and it showed that the trends of France, Germany, United Kingdom, the United States, and Spain stayed steady, while the trends of newly confirmed cases in Korea, Italy, and Iran laid between Hubei and non-Hubei, which have been identified as the major epidemic areas in the globe (Fig. 4b and c).

Then the established STM model was implemented to the three countries. The results showed that the IFP in South Korea would arrive from Mar 6 to 12, 2020 (Fig. 5a and b); the IFP in Italy would arrive from Mar 10 to 24, 2020 (Fig. 5c and d); the IFP in Iran would come from Mar 10 to 24, 2020 (Fig. 5e and f). After completing the model and training set establishment, we compared the cumulative case prediction with the actual data on Mar
6 and Mar 9, which was validation set, and the results overtly testified the efficacy of this prediction model all in Korean, Italy, and Iran (Table 3). By utilizing this model, the approximate number of confirmed cases in the three countries at the end of March, April, and May could be predicted (details show in Fig. 6), which could instruct the international medical resources allocation.

\section{The verification of STM model by the data updating after prediction}

With the time going by almost 3 months, we reran the STM model in South Korea, Italy, and Iran. The results showed that the STM model well predicted the trend in South Korea and Italy, however, not in Iran (Fig. 7a to f). In South Korea, the line of the total confirmed cases laid between the optimistic scenario and cautiously optimistic scenario before April, and fitting the cautiously optimistic scenario after that (Fig. 7a), and so did the IFP of the confirmed cases in South Korea (Fig. 7b). In Italy, the line of the total confirmed cases fitting the relatively pessimistic scenario (Fig. 7c), and so did the IFP of Italy (Fig. 7d).

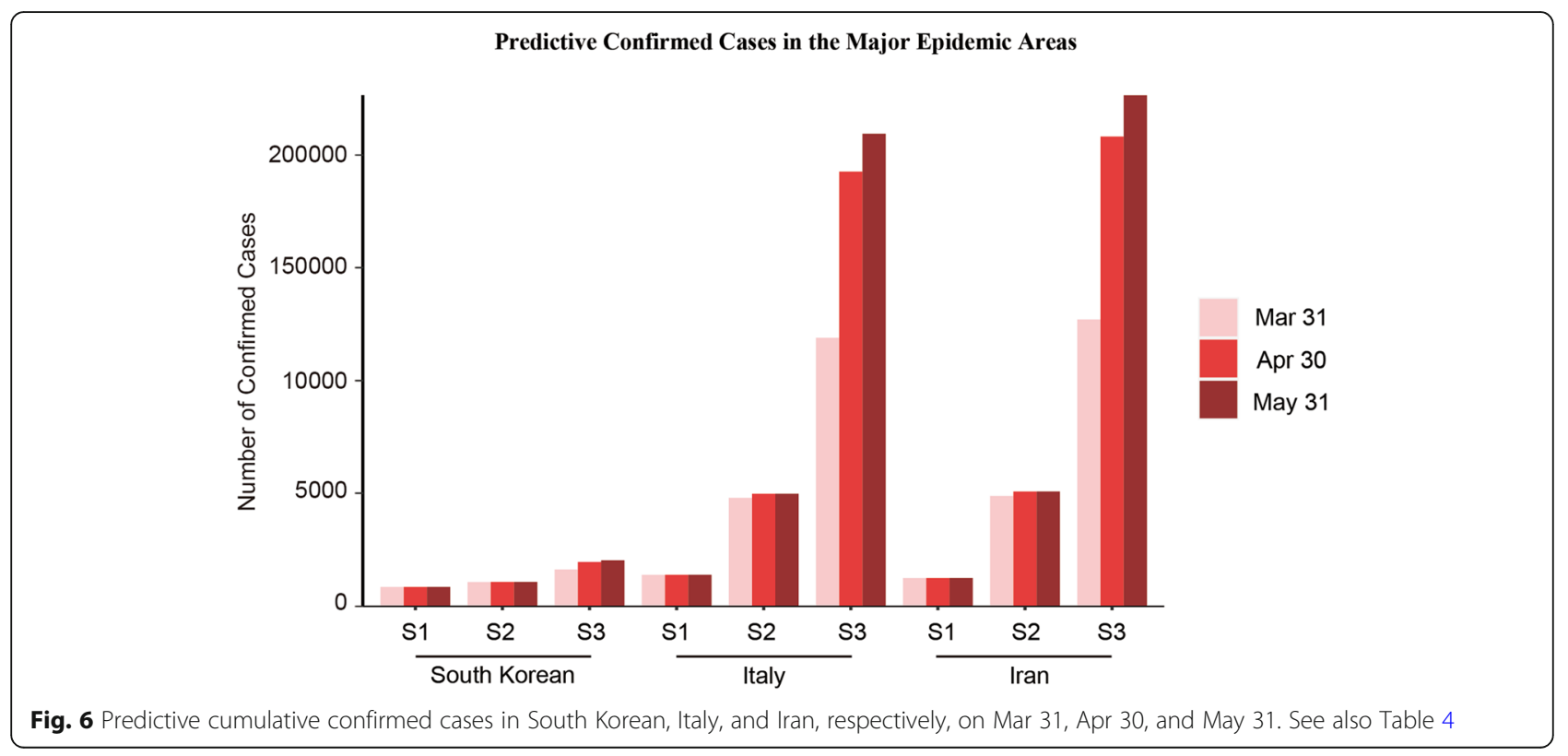


Table 4 Predictive Cumulative Confirmed Cases in the Major Epidemic Areas

\begin{tabular}{|c|c|c|c|c|c|c|c|c|c|}
\hline \multirow{2}{*}{$\begin{array}{l}\text { Country } \\
\text { Scenario }\end{array}$} & \multicolumn{3}{|c|}{ South Korean } & \multicolumn{3}{|l|}{ Italy } & \multicolumn{3}{|l|}{ Iran } \\
\hline & $\mathrm{S1}$ & S2 & S3 & $\mathrm{S1}$ & S2 & S3 & S1 & S2 & S3 \\
\hline $2020 / 3 / 31$ & 8539 & 10,651 & 16,168 & 13,797 & 47,930 & 118,878 & 12,339 & 48,847 & 126,978 \\
\hline $2020 / 4 / 30$ & 8541 & 10,695 & 19,454 & 13,815 & 49,797 & 192,593 & 12,353 & 50,795 & 208,006 \\
\hline $2020 / 5 / 31$ & 8541 & 10,695 & 20,198 & 13,815 & 49,802 & 209,272 & 12,353 & 50,800 & 226,340 \\
\hline
\end{tabular}

S1: optimistic scenario; S2: cautiously optimistic scenario; S3: relatively pessimistic scenario

Nevertheless, in Iran, the line of the total confirmed cases laid between the optimistic scenario and the relatively pessimistic scenario (Fig. 7e), and the increment of confirmed cases is still relatively high, which means the IFP of Iran has not come yet (Fig. 7f).

\section{Discussion}

The concept of state transfer matrix was put forward by Russian mathematician Markov. In the early twentieth century Markov found in the early twentieth century that for some factors of a system in the transfer, the result is only affected by the n-1 result, that is, it is only related to the current state, and has nothing to do with the past state. Thus in Markov analysis, the concept of state transition is introduced. The so-called state refers to the state in which objective things may appear or exist; State transition refers to the probability of objective things being transferred from one state to another. In this study, to estimate the IFP of new confirmed cases in the future and the global cumulative case size, we chose markov model cohort simulation. We define the different course states of COVID-19 as state vectors, through which state vectors are used to identify the state of close contacts or patients, and apply line equations to deduce the state transfer equation under $\mathrm{N}$ cycles. Using this STM model, we were able to predict the arrival date of IFP for newly confirmed cases in Hubei or non-Hubei provinces, and set three preset scenarios from China to match and fit major endemic areas, including Korea, Italy, and Iran, in order to control model errors.

In this study, the STM model of Korea, Italy and Iran was established in March, and the model data was continuously updated in the background daily. So far, the number of confirmed cases in the world has soared to more than 8 million [15]. As shown in Fig. 7, we successfully predicted the trend of the outbreak in South Korea and Italy, but Iran did not meet expectations. Considering that South Korea and Italy formed good medical experience exchange and medical resources support with Chinese medical experts at the early stage of the outbreak, it is understandable that the model based on Chinese data has a good prediction of the development trend of the epidemic in South Korea and Italy through the absorption of China's experience and response mode. In the Lancet's the Healthcare Access and
Quality Index for 195 Countries and Territories and Selected Subnational Locations, Italy, South Korea and China all reach a good score, which can also be seen as having comparable national healthcare and epidemic prevention and control capabilities. The successful prediction of South Korea and Italy can prove that the development trend of newly and accumulatively diagnosed patients is consistent with the prediction of this model and changes according to our preset prediction model when medical resources are guaranteed and diagnostic capacity is sound. The model in this study did not predict the trend of the outbreak in Iran as expected. The main reason may be that Iran's diagnostic capacity is limited by the unreasonable allocation of international medical resources, the shortage of PCR kits and the lack of CT scanners. The presence of people with the virus, those with atypical symptoms, and those with mild and asymptomatic symptoms reveals a huge risk of missed diagnosis and recurrence of outbreaks due to inadequate diagnostic conditions [16-18]. In general, the model successfully predicted the outbreak trend of major covid-19 developing countries in the first half of 2020, and had a high guidance effect on the allocation of international medical resources during the epidemic.

Given the punchy transmissibility of COVID-19 [19], isolation and quarantine are undoubtedly the primary options [11]. And currently, predicting models of built for epidemic sprouted out a lot. Ziff et al. established a model of death cases and reported that death cases follow three patterns: exponential growth, power-law behavior, and then exponential decline in the daily rate [20]. Nevertheless, deaths are affected by many factors, such as age [21, 22]. More attention should be paid to the number of new cases, and the rate of increment, attributed to the effect of epidemic prevention and control, can be evaluated to guide the date of return to work. Based on the epidemiological data of 186 countylevel administrative units in the UK, Davies et al. established a random inter-compartmental model, in which individuals were divided into susceptibility, exposure, infection (preclinical, clinical, or subclinical) and recovery status (removed from the model). The model is stratified by the age of 5 years, and the impact of various basic interventions on R0 is evaluated [23]. Scheiner et al. adjusted the classic epidemiological model, i.e., SEIR 
A South Korea: Total Confirmed Infection Cases

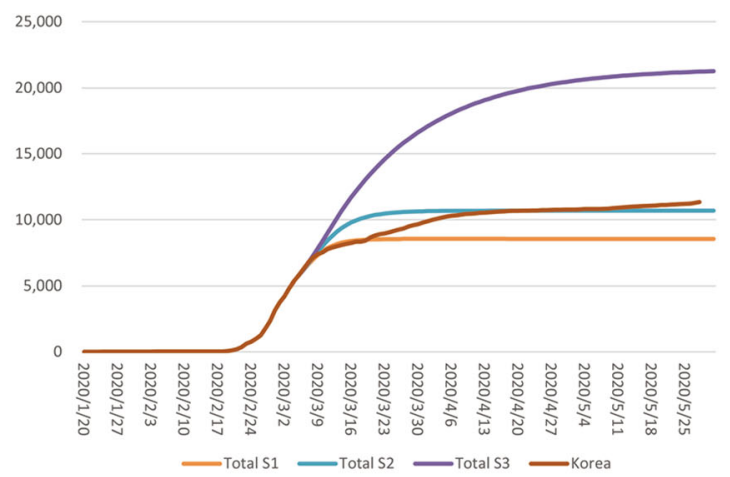

C

Italy: Total Confirmed Infection Cases

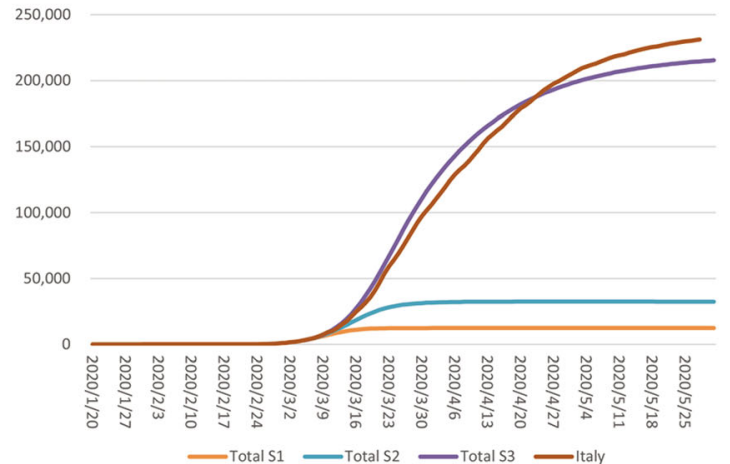

$\mathbf{E}$

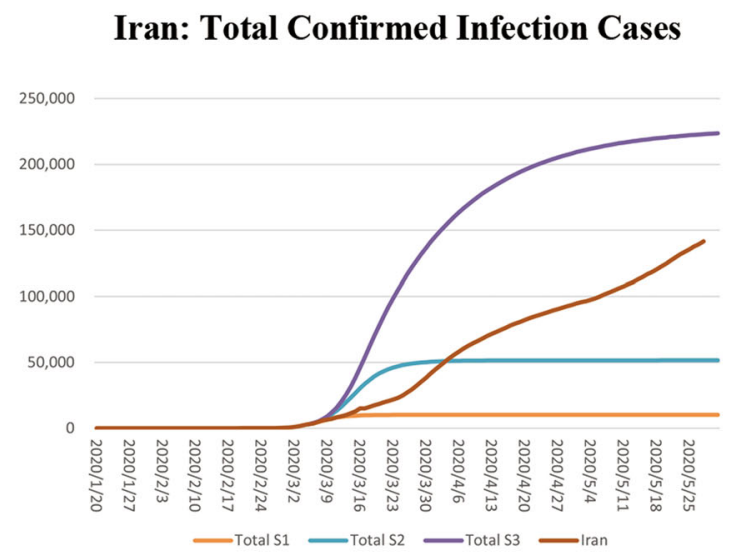

B South Korea: Increment of Confirmed Cases

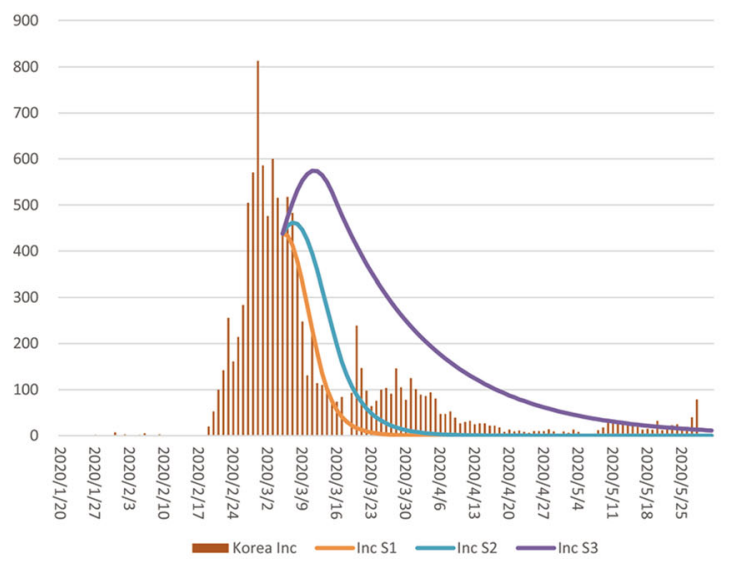

D

Italy: Increment of Confirmed Cases

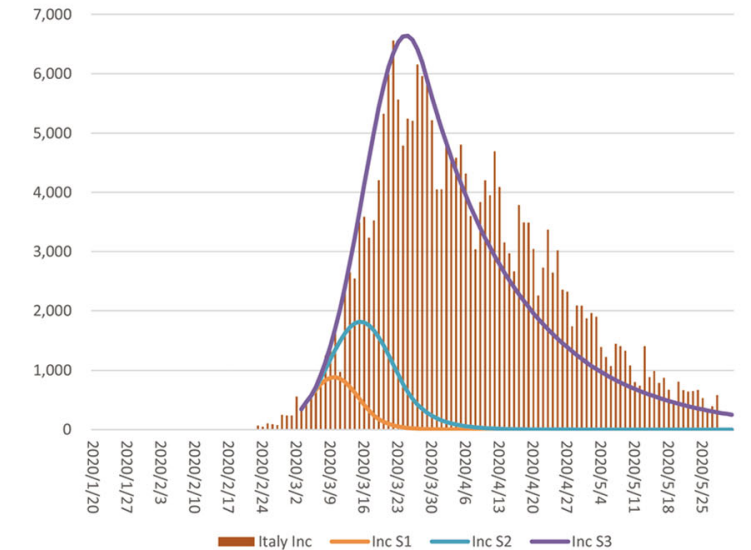

F

Iran: Increment of Confirmed Cases

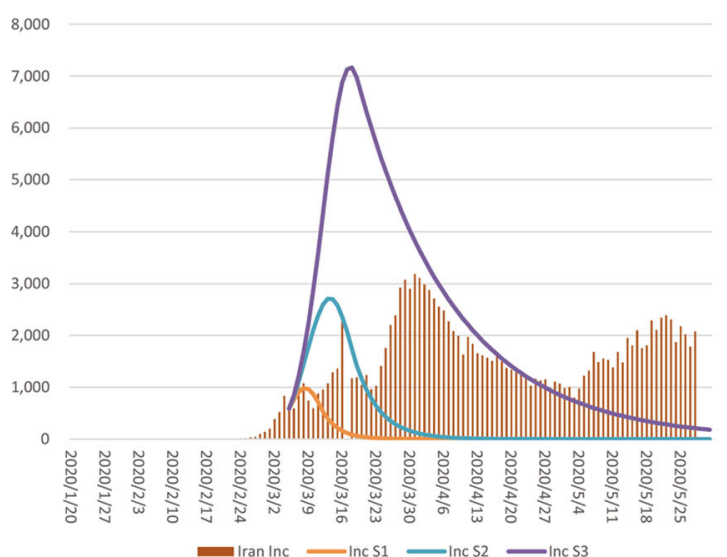

Fig. 7 Model verification in South Korea, Italy, and Iran. a-f Predictive total confirmed cases and increment of confirmed cases in South Korean, Italy, and Iran, respectively, with the three scenarios deduced by the State Transition Matrix Model based on the data before Mar 6, 2020 and the true number of confirmed cases and increment in the three countries 
model. It's based on the transmission characteristics of coronavirus, and concluded that the rule of delay from infection to death was more representative of the actual situation than the classical death dynamics rule, so the traditional SEIR model could be more applicable to the prediction of the transmission of COVID-19 epidemic [24]. Hasan et al. proposed a hybrid model of integrated empirical mode decomposition (EEMD) and artificial neural network (ANN) to predict COVID-19 outbreaks, using window period real-time COVID-19 time series data from 22 January 2020 solstice on 18 May 2020. EEMD is used to decompose the time series data, generate sub-signals, denoise the original data, establish neural network structure to train the de-noised data, and obtain a prediction model superior to the traditional statistical analysis [25]. Tuli et al. use machine learning (ML) and cloud computing to track disease and predict epidemic growth, and deploy an improved model based on MLS on cloud computing platforms to more accurately predict epidemic growth behavior in real time [26].

Moreover, we must strictly follow the coping strategy and learn the Chinese model for dealing with NCP outbreaks. Li et al. developed a simple regression model, and based on this model, they estimated that about 34 founder patients outside of China were not observed in the early stage of transmission, and the global trend approximated an exponential increase, tenfold increase in 19 days [27]. This study reproduced the initial spreading mode to the world, yet made no prediction for the future trend, and exponential growth will be curbed immediately after the attention of local governments, and the IFP will come. Milan Batista proposed an estimate of the final size of the COVID-19 epidemic, the logistic growth model and classic susceptible-infected-recovered dynamic model are used to estimate the final size of the coronavirus epidemic, being approximately 83,700 ( \pm 1300) cases and that the peak of the epidemic was on Feb 92,020 [28]. However, as of Mar 5, the number of global cases has reached 95,333, and the IFP for growth in South Korea, Italy, and Iran has not yet arrived, which means the global size will be even more colossal.

Our model is based on the fitting of real data from standard authorities. Through the STM Model, based on data from Hubei and non-Hubei, we predict the IFPs in Korea, Italy, and Iran, while there are still some limitations. Due to the large outbreaks started at different times lines all over the world, the effects of seasonal and geographical factors have not been taken into account. Although the fitting with the Chinese model can better predict the situation around the world, through reference and learning, the response strategies of other countries may be more mature. As China resumes work, the production capacity of various medical resources will gear up rapidly, which will impose a positive impact on the world, and it could be more optimistic that the IFP will come soon.

Local governments, regardless of the speed of outbreaks, should learn from China's primary response strategy, such as stopping working, reducing gathering, preventing contact transmission, wearing masks, and implementing quarantine. After the NCP being under control, the production and output of medical resources should be intensified, the production of coronavirus detection kits should be accelerated, existing cases should be summarized. More accurate diagnostic criteria should be compiled to prevent massive missed diagnoses in countries lacking the kit. Even if it currently causes some global economic regression, the recovery will swiftly come after holding the throat of NCP and COVID-19.

\section{Conclusion}

Based on data from China, we utilized the State Transition Matrix Model to predict the IFP of disease in countries currently experiencing outbreaks worldwide. If properly controlled, the IFP in South Korea and Italy will come in early March, and the IFP in Iran will come in mid-March. And through almost 3 months, our model fitted well in South Korea and Italy, however, not Iran, partly because of the irrational international medical resource allocation. During this period, countries around the world should work together to fight the epidemic.

\section{Abbreviations \\ NCP: Novel coronavirus pneumonia; COVID-19: Coronavirus disease 2019; COV: Coronavirus; SARS: Severe acute respiratory syndrome; \\ ACE2: Angiotensin-converting enzyme 2; IFP: Inflection point; HCHP: The Health Commission of Hubei Province; NHC: The National Health \\ Commission of the People's Republic of China; WHO: The World Health \\ Organization; STM: State transition matrix; MO: Medical observation; Disc: Discharge; NS: Non-severe case; S: Severe case; Cr: Critical case; Cu: Cured case; D: Death; V: Vector; NCC: New close contacts; 5DMA: 5-day moving average; 10DMA: 10-day moving average; SEIR: susceptible - exposed - infectious - recovered; EEMD: Empirical mode decomposition; ANN: Artificial neural network; ML: Machine learning}

\section{Acknowledgements \\ We acknowledge the support from Youth Science and Technology Innovation Studio of Shanghai Jiao Tong University School of Medicine.}

\section{Authors' contributions}

$J C$ and JZ made substantial contributions to the conception and design of the work; ZZ and KW performed acquisition, interpretation and analysis of data; $Z Y$ and $X Z$ drafted the manuscript. All author have provided critical review of the manuscript and approved the submitted version. All author have agreed both to be personally accountable for the author's own contributions and to ensure that questions related to the accuracy or integrity of any part of the work, even ones in which the author was not personally involved, are appropriately investigated, resolved, and the resolution documented in the literature.

\section{Funding}

The reported work was supported in part by research grants from the National Natural Science Foundation of China (no. 81972393, 81772705, 31570775). The funders had no role in the design and conduct of the study; collection, management, analysis, and interpretation of the data; preparation, review, or approval of the manuscript; and decision to submit the manuscript for publication. 


\section{Availability of data and materials}

The datasets used and analyzed during the current study are available from the corresponding author on reasonable request.

\section{Ethics approval and consent to participate}

There is not patient or animal who participated in this study, and informed consent was not applicable. This study was approved by the Ethical Committee of Shanghai Jiao Tong University according to the Chinese Ethical Regulations.

\section{Consent for publication}

Not applicable.

\section{Competing interests}

The authors declare that they have no competing interests.

\section{Author details}

'Department of Urology, Shanghai General Hospital, Shanghai Jiao Tong University, School of Medicine, Shanghai, China. ${ }^{2}$ Department of Pharmacy, Huashan Hospital, Fudan University, Shanghai, China. ${ }^{3}$ School of Pharmacy, Fudan University, Shanghai, China. ${ }^{4}$ CreditWise Technology Company Limited, Floor 4-5, Section B, Building 1 Tianfu 5th Ave., Chengdu Hi-tech Zone, Chengdu 610041, China. ${ }^{5}$ Caixin Insight Group, Beijing, China. ${ }^{6} \mathrm{MSCl}$, Shanghai, China.

Received: 29 March 2020 Accepted: 14 September 2020

Published online: 29 September 2020

\section{References}

1. Li Q, et al. Early transmission dynamics in Wuhan, China, of novel coronavirus-infected pneumonia. N Engl J Med. 2020;382:1199-207.

2. NHC, Update on the epidemic situation of new coronavirus pneumonia as of 24:00 on March 9. National Health Commission of the People's Republic of China. http://www.nhc.gov.cn/xcs/yqtb/202003/948a03ad76f54d3583a01 8785efd7be9.shtml (Accessed 10 Mar 2020).

3. WHO, Novel coronavirus (2019-nCoV) situation report 49. World Health Organization, 2020. https://www.who.int/docs/default-source/coronaviruse/ situation-reports/20200309-sitrep-49-covid-19.pdf?sfvrsn=70dabe61_4 (Accessed 09 Mar 2020).

4. Holshue ML, et al. First case of 2019 novel coronavirus in the United States. N Engl J Med. 2020;382(10):929-36.

5. WHO, Novel coronavirus (2019-nCoV) situation report 1. World Health Organization, 2020. https://www.who.int/docs/default-source/coronaviruse/ situation-reports/20200121-sitrep-1-2019-ncov.pdf?sfvrsn=20a99c10_4 (Accessed 21 Jan 2020).

6. WHO, Novel coronavirus (2019-nCoV) situation report 45. World Health Organization, 2020. https://www.who.int/docs/default-source/coronaviruse/ situation-reports/20200305-sitrep-45-covid-19.pdf?sfvrsn=ed2ba78b_4. Accessed 5 Mar 2020

7. Tian X, et al. Potent binding of 2019 novel coronavirus spike protein by a SARS coronavirus-specific human monoclonal antibody. Emerg Microbes Infect. 2020;9(1):382-5.

8. Rothe C, et al. Transmission of 2019-nCoV infection from an asymptomatic contact in Germany. N Engl J Med. 2020;382(10):970-1.

9. Liu J, et al. Community Transmission of Severe Acute Respiratory Syndrome Coronavirus 2, Shenzhen, China, 2020. Emerg Infect Dis. 2020;26(6):1320-3.

10. Wang $M$, et al. Remdesivir and chloroquine effectively inhibit the recently emerged novel coronavirus (2019-nCoV in vitro. Cell Res. 2020:30(3):269-71.

11. Wilder-Smith A, Freedman DO. Isolation, quarantine, social distancing and community containment: pivotal role for old-style public health measures in the novel coronavirus (2019-nCoV) outbreak. J Travel Med. 2020;27(2): taaa020.

12. Wu K, Zheng J, Chen J. Utilize State Transition Matrix Model to Predict the Novel Corona Virus Infection Peak and Patient Distribution. medRxiv. 2020; p. 2020.02.16.20023614. https://doi.org/10.1101/2020.02.16.20023614.

13. Wu T, et al. Open-source analytics tools for studying the COVID-19 coronavirus outbreak. medRxiv. 2020; p. 2020.02.25.20027433. https://doi. org/10.1101/2020.02.25.20027433.

14. NHC, Update on the epidemic situation of new coronavirus pneumonia as of 24:00 on March 5. National Health Commission of the People's Republic of China. http://www.nhc.gov.cn/yjb/s7860/202003/b59dbcc84ed1498292 $714975039 d c d c 9 . s h t m l$ (Accessed 06 Mar 2020).

15. WHO, Novel coronavirus (2019-nCoV) situation report 150. World Health Organization, 2020. https:/www.who.int/docs/default-source/coronaviruse/ situation-reports/20200618-covid-19-sitrep-150.pdf?sfvrsn=aa9fe9cf_2 (19 June 2020).

16. Huang $L$, et al. Rapid asymptomatic transmission of COVID-19 during the incubation period demonstrating strong infectivity in a cluster of youngsters aged 16-23 years outside Wuhan and characteristics of young patients with COVID-19: A prospective contact-tracing study. J Infect. 2020; 80(6):e1-e13.

17. Rivett $L$, Sridhar S, Sparkes D, et al. Screening of healthcare workers for SARS-CoV-2 highlights the role of asymptomatic carriage in COVID-19 transmission. Elife. 2020;9:e58728. https://doi.org/10.7554/eLife.58728.

18. Wong MC, et al. Strengthening early testing and surveillance of COVID-19 to enhance identification of asymptomatic patients. J Infect. 2020;81(2): e112-3.

19. Chen TM, et al. A mathematical model for simulating the phase-based transmissibility of a novel coronavirus. Infect Dis Poverty. 2020:9(1):24.

20. Ziff AL, Ziff RM. Fractal kinetics of COVID-19 pandemic. medRxiv. 2020; p. 2020.02.16.20023820. https://doi.org/10.1101/2020.02.16.20023820.

21. Patel A, Jernigan DB. Initial public health response and interim clinical guidance for the 2019 novel coronavirus outbreak - United States, December 31, 2019-February 4, 2020. MMWR Morb Mortal Wkly Rep. 2020; 69(5): $140-6$

22. Wang W, Tang J, Wei F. Updated understanding of the outbreak of 2019 novel coronavirus (2019-nCoV) in Wuhan, China. J Med Virol. 2020;92(4): $441-7$.

23. Davies, N.G., et al., Effects of non-pharmaceutical interventions on COVID-19 cases, deaths, and demand for hospital services in the UK: a modelling study. Lancet Public Health, 2020;5(7):e375-e385.

24. Scheiner S, Ukaj N, Hellmich C. Mathematical modeling of COVID-19 fatality trends: death kinetics law versus infection-to-death delay rule. Chaos Solitons Fractals. 2020;136:109891

25. Hasan N. A methodological approach for predicting COVID-19 epidemic using EEMD-ANN hybrid model. Internet Things. 2020;11:100228.

26. Tuli S, et al. Predicting the growth and trend of COVID-19 pandemic using machine learning and cloud computing. Internet Things. 2020;11:100222.

27. Li Y, et al. COVID-19 Epidemic Outside China: 34 Founders and Exponential Growth. medRxiv. 2020; p. 2020.03.01.20029819. https://doi.org/10.1101/ 2020.03.01.20029819

28. Batista M. Estimation of the final size of the COVID-19 epidemic. medRxiv. 2020; p. 2020.02.16.20023606. https://doi.org/10.1101/2020.05.22.20110064

\section{Publisher's Note}

Springer Nature remains neutral with regard to jurisdictional claims in published maps and institutional affiliations.

Ready to submit your research? Choose BMC and benefit from:

- fast, convenient online submission

- thorough peer review by experienced researchers in your field

- rapid publication on acceptance

- support for research data, including large and complex data types

- gold Open Access which fosters wider collaboration and increased citations

- maximum visibility for your research: over $100 \mathrm{M}$ website views per year

At BMC, research is always in progress.

Learn more biomedcentral.com/submission 